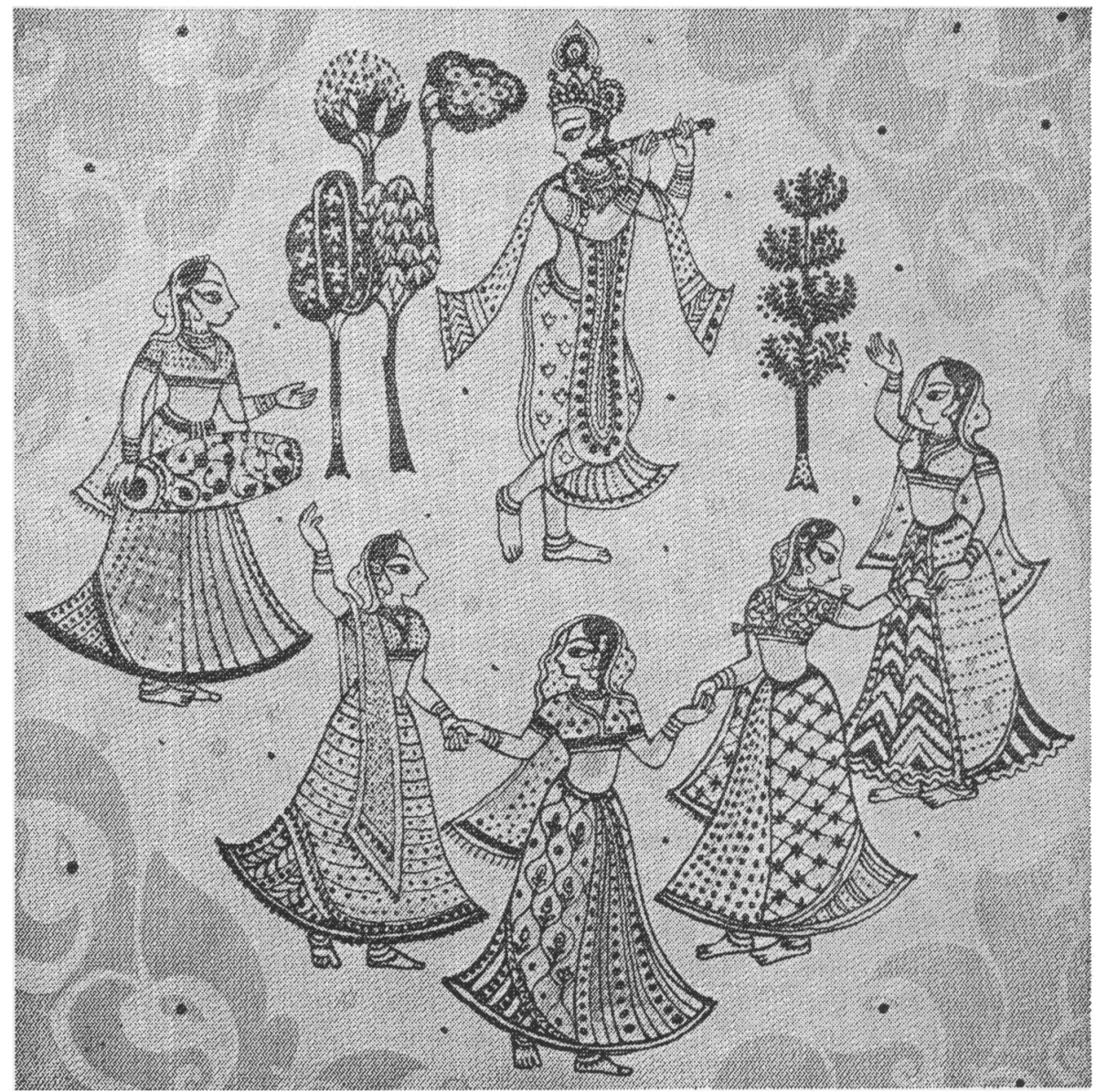

\title{
Love story
}

La magie, vous y croyez?

Alors regardez bien le décor de nos nouveaux Boeing 747 .

$\mathrm{Ce}$ décor raconte l'histoire de Krishna - la plus aimable, la plus humaine des incarnations de Vishnu.

II possédait sur les femmes un étrange et irrésistible pouvoir.

Où qu'il fôt, chaque fois qu'il appe- lait - les femmes accouraient.

Telles les belles de la peinture cidessus.

Pour les attirer, Krishna jouait de la flute.

Mais vous, il vous suffit d'appuyer sur un bouton. 
HOTEL INTERCONTINENTAL HOTEL INTERCONTINENTAL HOTEL INTERCONTI NENTAL HOTEL INTERCONTINENTAL HOTEL INTERCONTINENTAL HOTEL IN TERCONTINENTAL HOTEL INTERCONTINENTAL HOTEL INTERCONTINENTAL HOTEL INTERCONTINENTAL HOTEL INTERCONTINENTAL HOTEL INTERCONTI NENTAL HOTEL INTERCONTINENTAL HOTEL INTERCONTINENTAL HOTEL IN TERCONTINENTAL HOTEL INTERCONTINENTAL HOTEL INTERCONTINENTAL HOTEL INTERCONTINENTAL HOTEL INTERCONTINENTAL HOTEL INTERCONTI NENTAL HOTEL INTERCONTINENTAL HOTEL INTERCONTINENTAL HOTEL IN TERCONTINENTAL HOTEL INTERCONTINENTAL HOTEL INTERCONTINENTAL HOTEL INTERCONTINENTAL HOTEL INTERCONTINENTAL HOTEL INTERCONTI NENTAL HOTEL INTERCONTINENTAL HOTEL INTERCONTINENTAL HOTEL IN TERCONTINENTAL HOTEL INTERCONTINENTAL HOTEL INTERCONTINENTAL HOTEL INTERCONTINENTAL HOTEL INTERCONTINENTAL HOTEL INTERCONTI NENTAL HOTEL INTERCONTINENTAL HOTEL INTERCONTINENTAL HOTEL IN TERCONTINENTAL HOTEL INTERCONTINENTAL HOTEL INTERCONTINENTAL HOTEL INTERCONTINENTAL HOTEL INTERCONTINENTAL HOTEL INTERCONTI NENTAL HOTEL INTERCONTINENTAL HOTEL INTERCONTINENTAL HOTEL IN TERCONTINENTAL HOTEL IN HOTEL INTERCONTINENTAL \& NENTAL HOTEL INTERCONTII TERCONTINENTAL HOTEL IN HOTEL INTERCONTINENTAL \& NENTAL HOTEL INTERCONTII TERCONTINENTAL HOTEL IN HOTEL INTERCONTINENTAL \& NENTAL HOTEL INTERCONTII TERCONTIN HOTEL INTI NENTAL HC TERCONTIN HOTEL INTE NENTAL HC TERCONTIN HOTEL INTI NENTAL HC TERCONTINENTAL HOTEL IN HOTEL INTERCONTINENTAL \& NENTAL HOTEL INTERCONTII TERCONTINENTAL HOTEL IN HOTEL INTERCONTINENTAL \& NENTAL HOTEL INTERCONTII TERCONTINENTAL HOTEL IN HOTEL INTERCONTINENTAL \& NENTAL HOTEL INTERCONTII I HOTEL INTERCONTINENTAL INENTAL HOTEL INTERCONTI ITERCONTINENTAL HOTEL IN I HOTEL INTERCONTINENTAL INENTAL HOTEL INTERCONTI ITERCONTINENTAL HOTEL IN I HOTEL INTERCONTINENTAL INENTAL HOTEL INTERCONTI ITERCONTINENTAL HOTEL IN NTINENTAL NTERCONTI I HOTEL IN GENEVE NTINENTAL NTERCONTI I HOTEL IN NTINENTAL NTER CONTI I HOTEL IN I HOTEL INTERCONTINENTAL INENTAL HOTEL INTERCONTI ITERCONTINENTAL HOTEL IN I HOTEL INTERCONTINENTAL INENTAL HOTEL INTERCONTI JTERCONTINENTAL HOTEL IN I HOTEL INTERCONTINENTAL INENTAL HOTEL INTERCONTI ITERCONTINENTAL HOTEL IN TERCONTINENTAL HOTEL INTERCONTINENTAL HOTEL INTERCONTINENTAL HOTEL INTERCONTINENTAL HOTEL INTERCONTINENTAL HOTEL INTERCONTI NENTAL HOTEL INTERCONTINENTAL HOTEL INTERCONTINENTAL HOTEL IN TERCONTINENTAL HOTEL INTERCONTINENTAL HOTEL INTERCONTINENTAL HOTEL INTERCONTINENTAL HOTEL INTERCONTINENTAL HOTEL INTERCONTI NENTAL HOTEL INTERCONTINENTAL HOTEL INTERCONTINENTAL HOTEL IN TERCONTINENTAL HOTEL INTERCONTINENTAL HOTEL INTERCONTINENTAL HOTEL INTERCONTINENTAL HOTEL INTERCONTINENTAL HOTEL INTERCONTI NENTAL HOTEL INTERCONTINENTAL HOTEL INTERCONTINENTAL HOTEL IN TERCONTINENTAL HOTEL INTERCONTINENTAL HOTEL INTERCONTINENTAL HOTEL INTERCONTINENTAL HOTEL INTERCONTINENTAL HOTEL INTERCONTI NENTAL HOTEL INTERCONTINENTAL HOTEL INTERCONTINENTAL HOTEL IN TERCONTINENTAL HOTEL INTERCONTINENTAL HOTEL INTERCONTINENTAL HOTEL INTERCONTINENTAL HOTEL INTERCONTINENTAL HOTEL INTERCONTI NENTAL HOTEL INTERCONTINENTAL HOTEL INTERCONTINENTAL HOTEL IN TERCONTINENTAL HOTEL INTERCONTINENTAL HOTEL INTERCONTINENTAL HOTEL INTERCONTINENTAL HOTEL INTERCONTINENTAL HOTEL INTERCONTI NENTAL HOTEL INTERCONTINENTAL HOTEL INTERCONTINENTAL HOTEL IN TERCONTINENTAL HOTEL INTERCONTINENTAL HOTEL INTERCONTINENTAL HOTEL INTERCONTINENTAL HOTEL INTERCONTINENTAL HOTEL INTERCONTI 


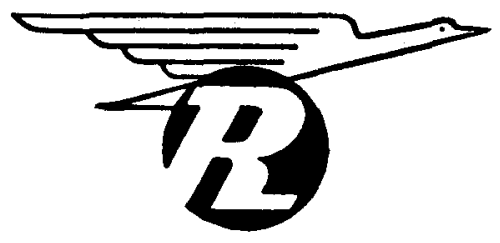

H. Aitscharal \& Cie.S.A. TRANSPORTS INTERNATIONAUX

\section{AGENCE DE VOYAGES}

GENEVE, 49, route des Jeunes

Téléphone 437600 - Télex 22167

Change - Billets - Passages maritimes

Assurances - Agence en douane

Camionnages - Entrepôts

Livraison d domicile des billets avion et chemin de fer sur simple commande téléphonique

Succursales:

LAUSANNE - ANNEMASSE (France) 


\section{Avec les compliments de}

\section{Vifor S.A.}

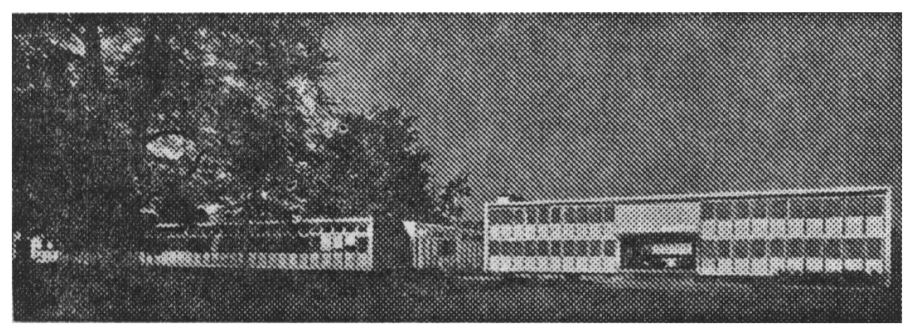

\section{Produits}

pharmaceutiques

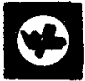




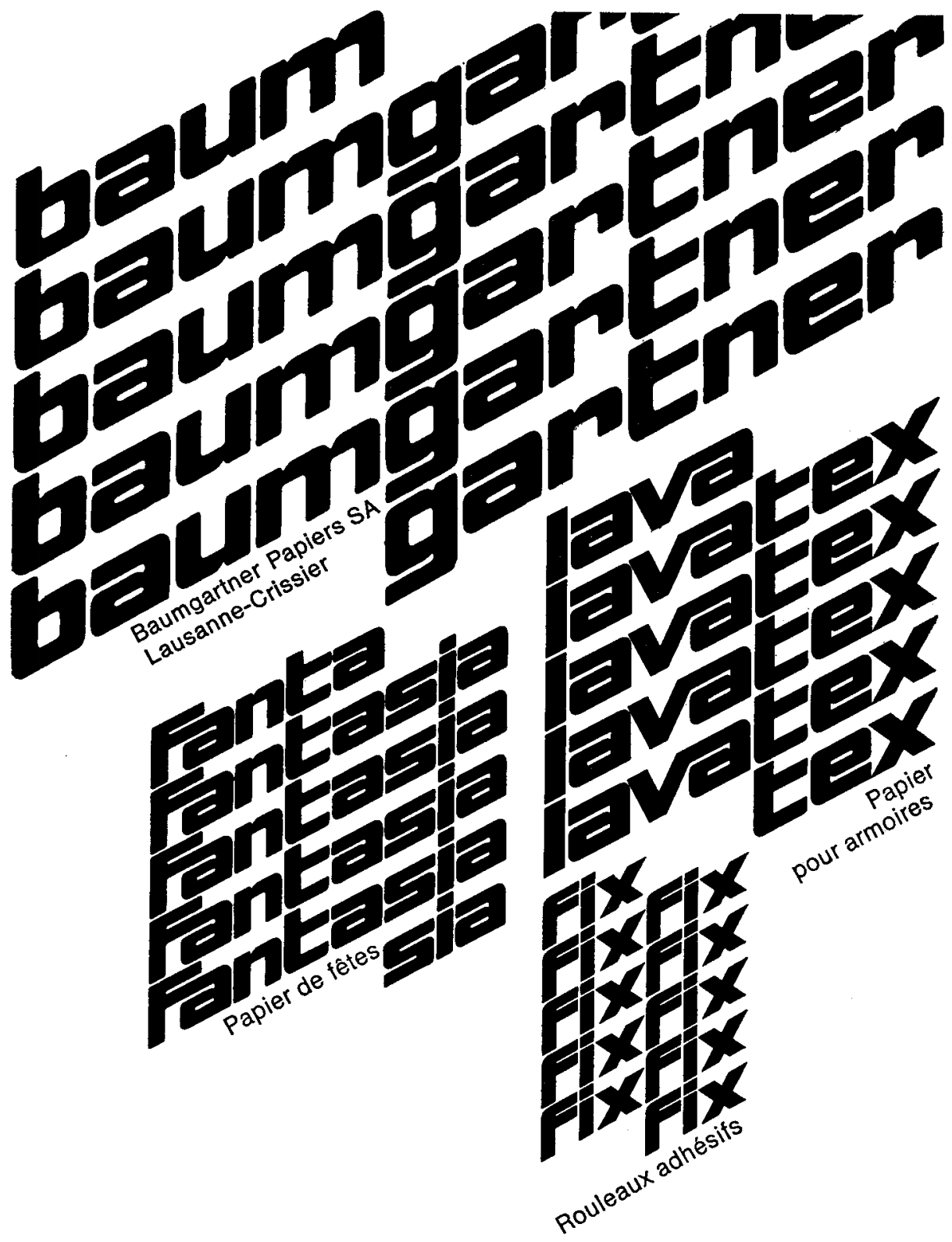




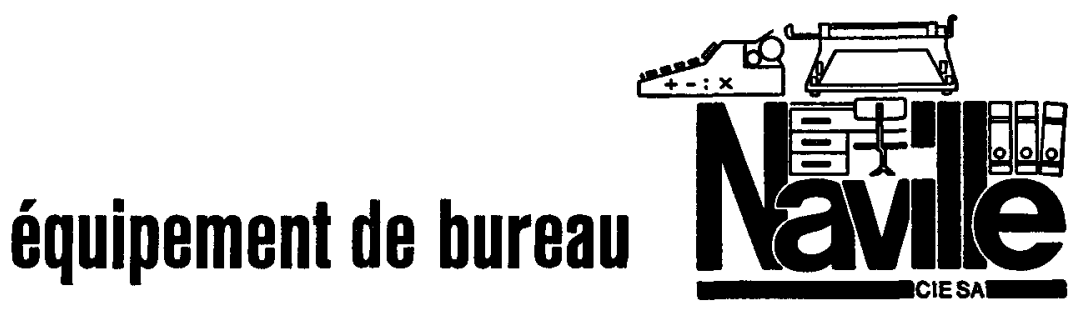

Meubles de bureau en bois et en acier Machines à écrire Calculatrices électroniques Appareils à dicter Philips Toutes les fournitures de bureau

GENĖVE 5-7, rue Lévrier (téléphone 322400 )

LAUSANNE 18, rue de Bourg (téléphone 22 82 33)

\section{Spécialités pharmaceutiques}

\section{Geistlich}

Spécialités d'action sûre et de conception moderne.

Ed. Geistlich Söhne A.G. Wohlhusen 


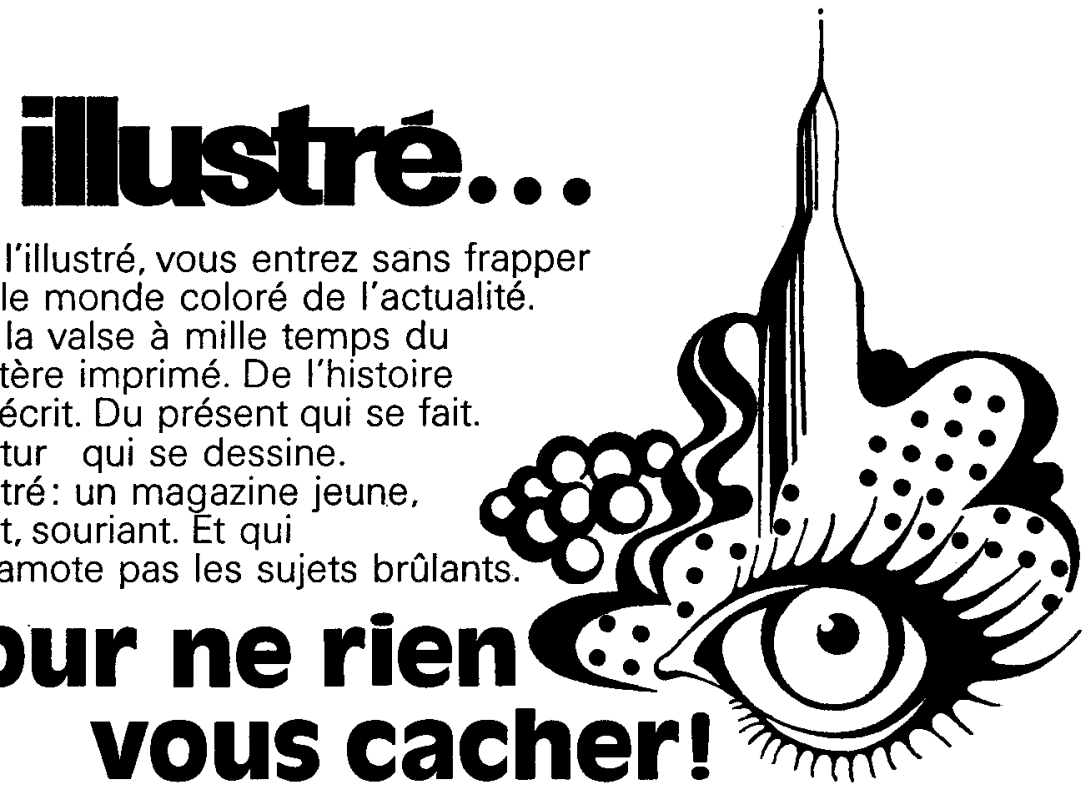

Avec l'illustré, vous entrez sans frapper dans le monde coloré de l'actualité. Dans la valse à mille temps du caractère imprimé. De l'histoire qui s'écrit. Du présent qui se fait. Du futur qui se dessine.

L'illustré: un magazine jeune. ouvert, souriant. Et qui n'escamote pas les sujets brûlants. pour ne rien
vous cacher!
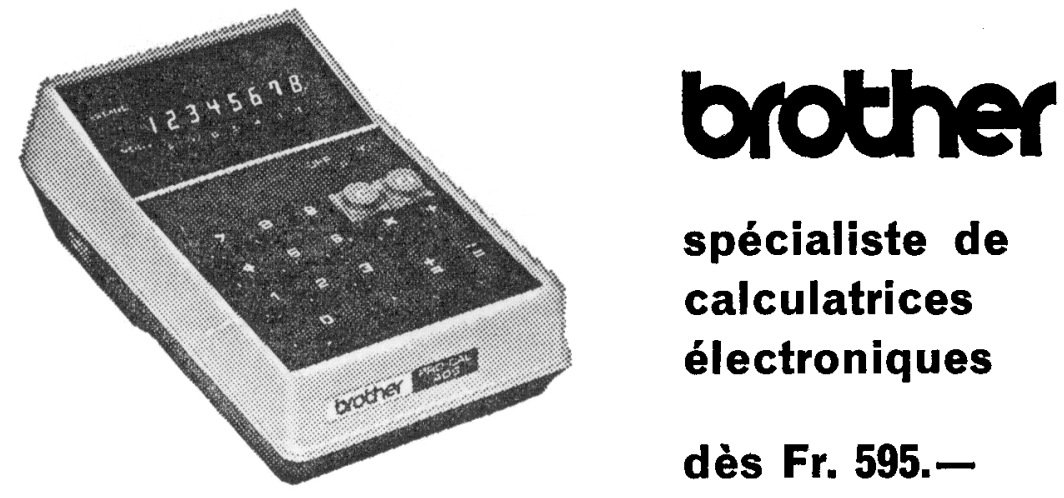

spécialiste de calculatrices électroniques

dès Fr. 595.-

atelier de réparations 

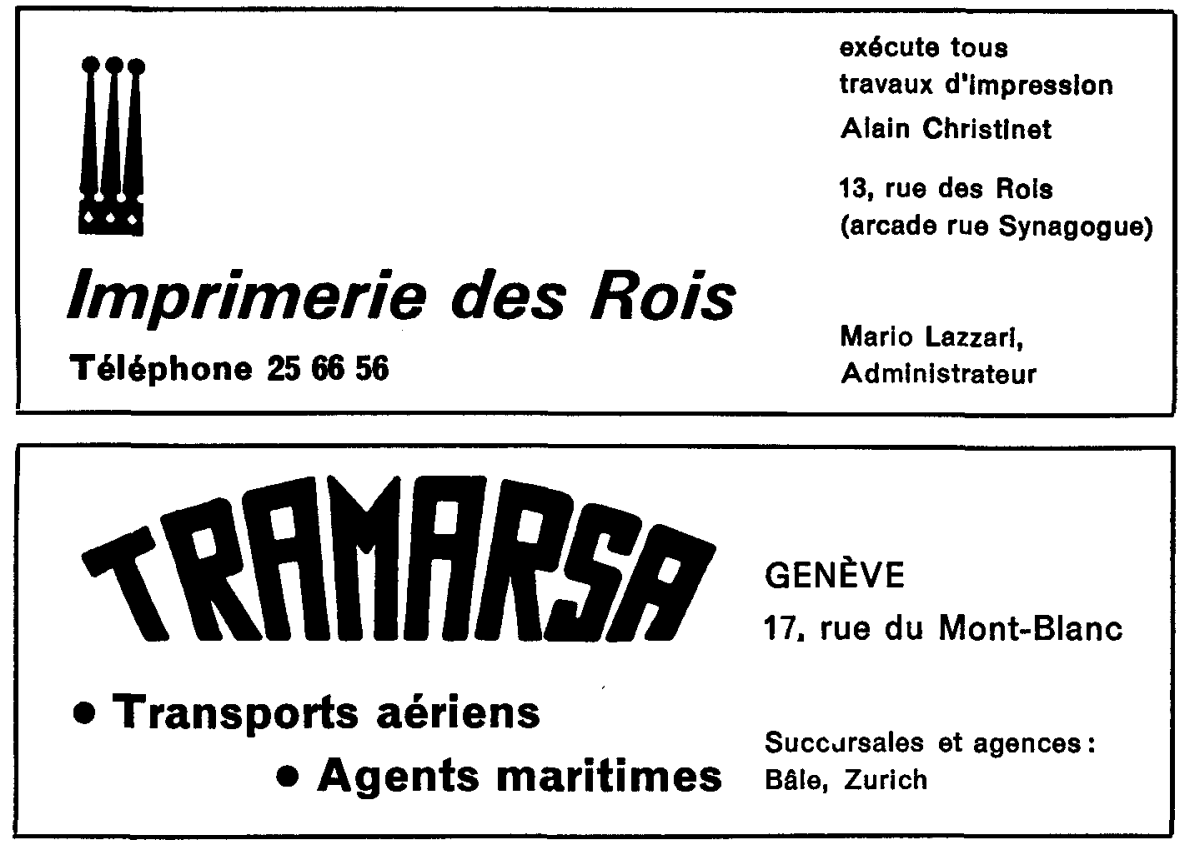

\section{clichéluth $*$ Fotolitio}

E. Lutz+Co. AG. 8005 Zürich Neugasse 116 Tel. 051/424211
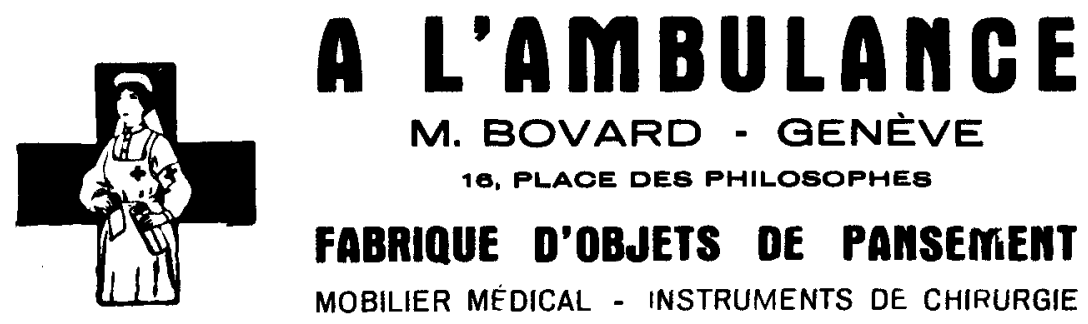

M. BOVARD - GENEVE 16. PLAOE des Philosophes

fabrique d'OBjets de Pahsement

MOBILIER MÉDICAL - INSTRUMENTS DE CHIRURGIE APPAREILS ELECTRO-MEDICAUX 


\section{EXTRAIT DES STATUTS \\ DU COMITÉ INTERNATIONAL DE LA CROIX-ROUGE \\ (adoptés le 25 septembre I952, révisés le 9 janvier 1964 et le 6 mai I97I)}

ARTICLE PREMIER. -- Le Comité international de la Croix-Rouge (CICR), fondé à Genève en I 863, consacré par les Conventions de Genève et par les Conférences internationales de la Croix-Rouge, est une institution indépendante ayant son statut propre.

Il est partie constitutive de la Croix-Rouge internationale ${ }^{1}$.

ART. 2 - En tant qu'association régie par les articles 60 et suivants du Code civil suisse, le CICR possède la personnalité civile.

ART. 3. - Le CICR a son siège à Genève.

Il a pour emblème la croix rouge sur fond blanc. Sa devise est "Inter arma caritas".

ART. 4. - Le CICR a, notamment, pour rôle:

a) de maintenir les principes fondamentaux de la Croix-Rouge, tels qu'ils ont été proclamés par la $\mathrm{XX}^{\mathrm{e}}$ Conférence internationale de la CroixRouge ;

b) de reconnaître toute Société nationale de la Croix-Rouge nouvellement créée ou reconstituée et répondant aux conditions de reconnaissance en vigueur, et de notifier cette reconnaissance aux autres Sociétés nationales;

c) d'assumer les tâches qui lui sont reconnues par les Conventions de Genève, de travailler à l'application fidèle de ces Conventions et de recevoir toute plainte au sujet de violations alléguées des Conventions humanitaires ;

d) d'agir, en sa qualité d'institution neutre, spécialement en cas de guerre, de guerre civile ou de troubles intérieurs; de s'employer en tout temps à ce que les victimes militaires et civiles desdits conflits et de leurs suites directes reçoivent protection et assistance, et de servir, sur le plan humanitaire, d'intermédiaire entre les parties;

e) de contribuer, en vue desdits conflits, à la préparation et au développement du personnel et du matériel sanitaires, en collaboration avec les organisations de la Croix-Rouge et les Services de santé militaires et autres autorités compétentes;

f) de travailler au perfectionnement du droit international humanitaire, à la compréhension et la diffusion des Conventions de Genève et d'en préparer les développements éventuels ;

g) d'assumer les mandats qui lui sont confiés par les Conférences internationales de la Croix-Rouge.

Le CICR peut en outre prendre toute initiative humanitaire qui entre dans son rôle d'institution spécifiquement neutre et indépendante et étudier toute question dont l'examen par une telle institution s'impose.

ART. 6 (alinéa premier). - Le CICR se recrute par cooptation parmi les citoyens suisses. Le nombre de ses membres ne peut dépasser vingt-cinq.

1 La Croix-Rouge internationale comprend les Sociétés nationales de la Croix-Rouge, le Comité international de la Croix-Rouge et la Ligue des Sociétés de la Croix-Rouge. L'expression \& Sociétés nationales de la Croix-Rouge, couvre également les Sociétés du Croissant-Rouge et la Société du Lion-et-Soleil-Rouge. 


\section{ADRESSES DES SOCIETES NATIONALES}

AFGHANISTAN - Croissant-Rouge afghan, Puli Artan, Kaboul

AFRIQUF DU SUD (République) - Croix-Rouge sud-africaine, Cor. Kruis \& Market Streets, P.O.B. 8726, Johannesburg.

ALBANIE - Croix-Rouge albanaise, 35, Rruga e Barrikadavet, Tirana.

ALGÉRIE - Comité central du Croissant-Rouge algérien, 15 bis, boulevard Mohamed V, Alger.

RÉPUBLIQUE DÉMOCRATIQUE ALLEMANDE - Croix-Rouge allemande dans la République démocratique allemande, 2 Kaitzerstrasse, Dx 801 Dresde l.

RÉPUBLIQUE FÉDÉRALE D'ALLEMAGNE Croix-Rouge allemande dans la République fédérale d'Allemagne, 71 , Friedrich-Ebert-Allee, 5300 Bonn 1, Postfach.

ARABIE SAOUDITE - Croissant-Rouge de l'Arabie Saoudite, Rivad.

ARGENTINF - Croix-Rouge argentine, H. Yrigoyen 2068, Buenos Aires.

AUSTRALIE - Croix-Rouge australienne, 122-128 Flinders Street, Melbourne, 3000 .

AUTRICHE - Croix-Rouge autrichienne, 3, Gusshausstrasse, Postfach 39, Vienne IV

BAHREIN - Croissant-Rouge de Bahrein, P.O. Box 882, Manama.

BELGIQUE - Croix-Rouge de Belgique, 98. chaussée de Vleurgat, 1050, Bruxelles.

BIRMANIE - Croix-Rouge de Birmanie, 42, Strand Road, Red Cross Building, Rangoon.

BOLIVIE - Croix-Rouge bolivienne, avenue SimonBolivar, 1515 (Casilla 741), La Paz.

BOTSWANA - Croix-Rouge du Botswana, Independence Avenue, P.O. Box 485. Gaberones.

BRESIL - Croix-Rouge brésilienne, Praça Cruz Vermelha, 10-12 Rio de Janeiro.

BULGARIE - Croix-Rouge bulgare, 1, boul. S. S. Biruzov, Sofia.

BURUNDI - Croix-Rouge du Burundi, B.P. 324, rue du Marché 3, Bujumhura.

CAMEROUN - Comité central de la Croix-Rouge camerounaise, rue Henri-Dunant, Boîte postale 631, Yaoundé.

CANADA - Croix-Rouge canadienne, 95, Wellesley Street East. Toronto, 284 (Ontario).

CHILI - Croix-Rouge chilienne, Avenida Santa Maria 0150 Correo 21, Casilla 246. V., Santiago de Chile.

CHINE - Croix-Rouge chinoise, 22, Kanmien Hutung, Pekin, E.

COLOMBIE - Croix-Rouge colombienne, Carrera 7a. N.॰ 34-65, Apartado Nacional 1110, Bogota D.E.

RÉPUBLIQUE DE CORÉE - Croix-Rouge de la République de Corée, 32-3ka, Nam San-Dong, Séoul.

RÉPUBLIQUE DÉMOCRATIQUE POPULAIRE DE CORÉE - Croix-Rouge de la République démocratique populaire de Corée, Pyongyang.

COSTA RICA - Croix-R ouge costaricienne Calle 5a, Apartado 1025, San José.
CÔTE D'IVOIRE - Croix-Rouge de Côte d'Ivoire, B.P. 1244, Abidian.

CUBA - Croix-Rouge cubaine, Calle 23201 esq. N Vedado, La Havane.

DAHOMEY - Société nationale de la Croix-Rouge du Dahomey, B.P. 1, Porto-Novo.

DANEMARK - Croix-Rouge danoise, Ny Vestergade 17, DK-1471 Copenhague $K$.

RÉPUBLIQUE DOMINICAINE - Croix-Rouge dominicaine, Calle Juan Enrique Dunant, Ensanche Miraflores, Apartado postal 1293, Saint-Domingue.

ÉGYPTE (République arabe) - Croissant-Rouge égyptien, rue Ramsès, 34, Le Caire.

ÉQUATEUR - Croix-Rouge équatorienne, Calle de la Cruz Roja y avenida Colombia 118, Quito.

ESPAGNE - Croix-Rouge espagnole, Eduardo Dato, 16, Madrid, 10.

ÉTATS-UNIS - Croix-Rouge américaine, National Headquarters, 17 th and D. Streets, N.W., Washington 20006, D.C.

ÉTHIOPIE - Croix-Rouge éthiopienne, Red Cross Road No 1, P.O. Box 195, Addis-Abéba.

FINLANDE - Croix-Rouge de Finlande. Tehtaankatu, 1 A, P.O.B. 14168, Helsinki 14.

FRANCE - Croix-Rouge française, 17, rue QuentinBauchart, F-75384 Paris, CEDEX 08.

GHANA - Croix-Rouge du Ghana, National Headquarters. Ministries Annex A3, P.O. Box 835, Accra.

GRÈCE - Croix-Rouge hellénique, Rue Lycavittou, 1, Athènes 135.

GUATEMALA - Croix-Rouge du Guatemala, $3^{\text {a }}$ Calle 8-40, Zona 1, Guatemala C.A.

GUYANE - Croix-Rouge de Guyane, P.O. Box 351, Eve Leary, Georgetown.

HAÏTI - Croix-Rouge haitienne, place des NationsUnies, B.P. 1337, Port-au-Prince.

HAUTE-VOLTA - Croix-Rouge voltaique, B.P. 340 , Ouagadougou.

HONDURAS - Croix-Rouge du Honduras, la Avenida Entre 3a y 4a Calles, No 313 Comayagüela D.C.

HONGRJE - Croix-Rouge hongroise, Arany Janos utca, 31, Budapest $V$.

INDE - Croix-Rouge de l'Inde, Red Cross Road 1, La Nouvelle-Delhi, $I$.

INDONÉSIE - Croix-Rouge indonésienne, Djalan Abdulmuis, 66, P.O. Box 2009. Djakarta.

IRAK - Croissant-Rouge de l'Irak, Al Mansour, Bagdad.

IRAN - Société du Lion-et-Soleil-Rouge de l'Iran, Avenue Ark, Téhéran.

IRLANDE - Croix-Rouge irlandaise, 16, Merrion Square, Dublin 2.

ISLANDE - Croix-Rouge islandaise, ldugatu 4, Post Box 872, Reykjavik.

ITALIE - Croix-Rouge italienne, 12, via Toscana, Rome. 
JAMAIIQUE - Croix-Rouge de la Jamaíque, 76, Arnold Road, Kingston 5.

JAPON - Croix-Rouge du Japon, 1-1-5, Shiba Daimon, Minato-Ku, Tokio 105.

JORDANIE - Croissant-Rouge jordanien, P.O. Box 10001, Amman.

KENYA - Croix-Rouge du Kenya, St. John's Gate, P.O. Box 40712, Nairobi.

RÉPUBLIQUE KHMĖRE - Croix-Rouge khmère,

17, Vithei Croix-Rouge khmère, B.P. 94, PhnomPenh.

KOWEÏT - Croissant-Rouge de Koweït, P.O. Box 1359, Koweit.

LAOS - Croix-Rouge lao, B.P. 650, Vientiane.

LESOTHO - Croix-Rouge du Lesotho, P.O. Box 366, Maseru.

LIBAN - Croix-Rouge libanaise, rue GénéralSpears, Beyrouth.

LIBÉRIA - Croix-Rouge du Libéria, National Headquarters, 107 Lynch Street P.O. Box 226, Monrovia.

RÉPUBLIOUE ARABE LIBYENNE - CroissantRouge libyen, Berka Omar Mukhtar Street, P.O. Box 541. Benghazi.

LIECHTENSTEIN - Croix-Rouge du Liechtenstein, FL-9490 Vaduz.

LUXEMBOURG - Croix-Rouge luxembourgeoise, Parc de la Ville, C.P. 1806, Luxembourg.

MADAGASCAR - Croix-Rouge de la République malgache, rue Clémenceau, B.P. 1168, Tananarive.

MALAISIE - Croix-Rouge de Malaisie, Jalan Belfield 519, Kuala Lumpur.

MALAWI - Croix-Rouge du Malawi, Hall Road, Blantyre. (P.O. Box 30080 Chichiri, Blantyre 3).

MALI - Croix-Rouge malienne, Route de Koulikora B.P. 280, Bamáko.

MAROC - Croissant-Rouge marocain, rue Benzakour, B.P. 189, Rabat.

MEXIQUE - Croix-Rouge mexicaine, Avenida Ejército Nacional N.0 1032, Mexico 10,DF.

MONACO - Croix-Rouge monégasque, bd de Suisse 27, Monte-Carlo.

MONGOLIE (Republique populaire de) - CroixRouge de la République populaire de Mongolie, Central Post Office Post Box 537, Oulan-Bator.

NÉPAL - Croix-Rouge du Népal, Tahachal, P.B. 217, Kathmandu.

NICARAGUA - Croix-Rouge du Nicaragua, 305, 12 Avenida Noroeste, Managua, D.N.

NIGER - Croix-Rouge du Niger, B.P. 386, Niamey.

NIGÉRIA - Croix-Rouge du Nigéria, Eko Aketa Close, off. St. Gregory Rd., Onikan, P.O. Box. 764, Lagos.

NORVĖGE - Croix-Rouge de Norvège, Parkveien, $33 \mathrm{~b}$, Oslo 2.

NOUVELLE-ZÉLANDE - Croix-Rouge néo-zélandaise, Red Cross House, 14, Hill Street, Wellington 1. (P.O. Box 12-140, Wellington North).

OUGANDA - Croix-Rouge de l'Ouganda, Nabunya Road, P.O. Box 494, Kampala.

PAKISTAN - Croix-Rouge du Pakistan, Dr. Dawood Pota Road, Karachi 4.

PANAMA - Croix-Rouge de Panama, Apartado postal 668, Zona 1, Panama.

PARAGUAY - Croix-Rouge paraguayenne, calle André Barbero y Artigas 33, Asuncion.

PAYS-BAS - Croix-Rouge néerlandaise, 27, Prinsessegracht, La Haye.

PÉROU - Croix-Rouge péruvienne, Jiron Chancay 881, Lima.
PHILIPPINES - Croix-Rouge philippine, 860, United Nations Avenue, P.O. Box 280, Manille $D-406$.

POLOGNE - Croix-Rouge polonaise, Mokotowska 14, Varsovie.

PORTUGAL - Croix-Rouge portugaise, Secrétariat général, Jardim 9 de Abril, 1-5, Lisbonne 3.

ROUMANIE - Croix-Rouge de la République Socialiste de Roumanie, Strada Biserica Amzei, 29, Bucarest.

ROYAUME-UNI - Croix-Rouge britannique, 9, Grosvenor Crescent, Londres, S.W.1X 7EJ.

SAINT-MARIN - Croix-Rouge de Saint-Marin, Palais gouvernemental, Saint-Marin.

SALVADOR, EL - Croix-Rouge du Salvador, $3^{\mathrm{a}}$ avenida Norte y $3^{\mathrm{a}}$, calle Poniente, 21, San Salvador.

SÉNÉGAL - Croix-Rouge sénégalaise, Bld. Franklin-Roosevelt. P.O.B. 299, Dakar.

SIERRA LEONE - Croix-Rouge de Sierra Leone, P.O. Box 427, 6, Liverpool Street, Freetown.

SOMALIE - (République de) Croissant-Rouge somalien P.O. Box 937, Mogadiscio.

SOUDAN - Croissant-Rouge soudanais, P.O. Box 235, Khartoum.

RÉPUBLIQUE DE SRI LANKA (CEYLAN) Croix-Rouge de la République de Sri Lanka 106, Dharmapala Mawatha, Colombo 7.

SUĖDE - Croix-Rouge suédoise, Artillerigatan, 6, S-114 51 Stockholm 14.

SUISSE - Croix-Rouge suisse. Taubenstrasse 8 , Case postale 2699, 3001 Berne.

SYRIE - Croissant-Rouge syrien, bd Mahdi Ben Barake, Damas.

TANZANIE - Croix-Rouge de Tanganyika, Upanga Road. P.O.B. 1133, Dar-es-Salaam.

TCHÉCOSLOVAQUIE - Croix-Rouge tchécoslovaque, Thunovska, 18, Prague $I$.

THAIILANDE - Croix-Rouge thaillandaise, King Chulalongkorn Memorial Hospital, Bangkok.

TOGO - Croix-Rouge togolaise, 51, rue Boko Soga, B.P 655, Lomé.

TRINITÉ-et-TOBAGO - Croix-Rouge de Trinitéet-Tobago, Regional Community Park, Wrightson Road Extension, P.O. Box 357, Port-of-Spain Trinidad, West Indies.

TUNISIE - Croissant-Rouge tunisien, 19, rue d'Angleterre, Tunis.

TURQUIE - Sociêté du Croissant-Rouge turc, Yenisehir, Ankara.

U.R.S.S. - Alliance des Sociétés de la Croix-Rouge et du Croissant-Rouge de l'U.R.S.S., Tcheremushki, I. Tcheremushkinskii proezd 5, Moscou, $B 36$.

URUGUAY - Croix-Rouge uruguayenne, Avenida 8 de Octubre 2990 , Montevideo.

VENEZUELA - Croix-Rouge vénézuélienne, Avenida Andrés Bello, № 4, Apart. 3185, Cararas.

RÉPUBLIQUE DÉMOCRATIQUE DU VIETNAM - Croix-Rouge de la République démocratique du Vietnam, 68, rue Bà-Triè, Honot.

RÉPUBLIQUE DU VIETNAM - Croix-Rouge de la République du Vietnam, rue Hông Thâp Tu, No $^{\circ} 201$, Saïgon.

YOUGOSLAVIE - Croix-Rouge de Yougoslavie, Simina ulica broj, 19. Belgrade.

ZAIIRE (République du) - Croix-Rouge de la République du Zaîre, 41, av. de la Justice B.P. 1712, Kinshasa.

ZAMBIE - Croix-Rouge de Zambie, P.O. Box R. W. 1, 2838 Brentwood Drive, Lusaka. 